\title{
Optical trap for an atom around the midpoint between two coupled identical parallel optical nanofibers
}

\author{
Fam Le Kien $\odot$, Síle Nic Chormaic, and Thomas Busch $\odot$ \\ Okinawa Institute of Science and Technology Graduate University, Onna, Okinawa 904-0495, Japan
}

(Received 28 April 2021; accepted 20 May 2021; published 11 June 2021)

\begin{abstract}
We study the trapping of a ground-state cesium atom in a small region around the midpoint between two coupled identical parallel optical nanofibers. We suggest using a blue-detuned guided light field in the odd $\mathcal{E}_{z}$-sine array mode to produce an optical potential with a local minimum of exactly zero at the midpoint between the nanofibers. We find that the effects of the van der Waals potential on the total trapping potential around the minimum point are not significant when the fiber separation distance and the power of the guided light field are large. For appropriate realistic parameters, a net trapping potential with a significant depth of about $1 \mathrm{mK}$, a large scattering-limited coherence time of several seconds, and a large recoil-heating-limited trap lifetime of several hours can be obtained. We investigate the dependencies of the trapping potential on the power of the guided light field, the fiber radius, the wavelength of light, and the fiber separation distance.
\end{abstract}

DOI: 10.1103/PhysRevA.103.063106

\section{INTRODUCTION}

Optical nanofibers are tapered fibers with a subwavelength diameter and significantly differing core and cladding refractive indices [1]. Optical nanofibers have been investigated for various applications in nonlinear optics, atomic physics, quantum optics, and nanophotonics [1-4]. In particular, nanofibers have been used for trapping and optically interfacing cold atoms with guided light fields [5-20].

A successful technique for trapping atoms near a nanofiber is to combine optical dipole forces of a blue- and a reddetuned guided light field $[5,6]$. This two-color trap scheme has been experimentally realized for laser-cooled alkali-metal atoms at about $200 \mathrm{~nm}$ from the nanofiber surface [7,8,1013]. Other waveguide-based atom traps have been proposed and investigated, such as a combination of the attractive potential of a red-detuned guided field and the repulsive potential of the centrifugal force [14], interference of higher-order modes $[15,16]$, a diffracted laser field impinging perpendicularly to the fiber [17], a helical two-color trapping potential [18], a combination of fictitious and real magnetic fields [19], a nanofiber with a slot [20,21], and an alligator photonic crystal waveguide [22-25].

Coupled waveguides play an important role in numerous optical devices such as multicore fibers, optical directional couplers, polarization splitters, ring resonators, and interferometers [26-28]. Recently, optical devices composed of twisted or knotted nanofibers have been fabricated [29]. Coupling between two nanofibers has been studied [29,30] in the framework of the coupled mode theory [26-28]. It has been shown that the guided normal (array) modes of two

Published by the American Physical Society under the terms of the Creative Commons Attribution 4.0 International license. Further distribution of this work must maintain attribution to the author(s) and the published article's title, journal citation, and DOI. coupled dielectric rods can be calculated by using the circular harmonics expansion [31]. This method has been extended to multicore fibers [32-35]. The propagation constant and the flux density of the field in a guided normal mode have been studied [31,35,36]. The polarization patterns [35] and the mode cutoffs [37] have been investigated. In optomechanics, forces arising from internal illumination by light traveling in coupled waveguides have been studied [38], and light-guiding arrays of mechanically compliant glass nanospikes have been fabricated [39].

Recently, the spatial distributions of the fields in the guided array modes of two coupled parallel nanofibers have been examined [40]. It has been shown that the distribution of the field intensity in the odd $\mathcal{E}_{z}$-sine array mode has a local minimum of exactly zero at the midpoint between the nanofibers [40]. This feature can be used to trap atoms with a single blue-detuned light field. In order to realize an optical trap for atoms in a small region around the midpoint between the nanofibers, we need to produce an optical potential that can dominate the surface-induced van der Waals potential in the trapping region. The parameters for the system must be realistic, while the characteristics of the obtained trapping potential must be appropriate for applications. The possibility of trapping atoms between two parallel nanofibers may open up new applications in nonlinear optics, quantum optics, and quantum information. While a setup with infinite, parallel nanofibers might be currently hard to realize, the configuration is also somewhat similar to a slotted fiber [20,21] or an alligator photonic crystal waveguide [22-25]. Therefore, it is desirable to study this issue in detail.

In this work, we study the possibility of trapping a groundstate cesium atom in a small region around the midpoint between two coupled identical parallel optical nanofibers. We investigate the dependencies of the trapping potential on the power of the guided light field, the fiber radius, the wavelength of light, and the fiber separation distance. We show that a blue-detuned far-off-resonance field in the odd $\mathcal{E}_{z}$-sine array mode can produce a trapping potential around the midpoint 


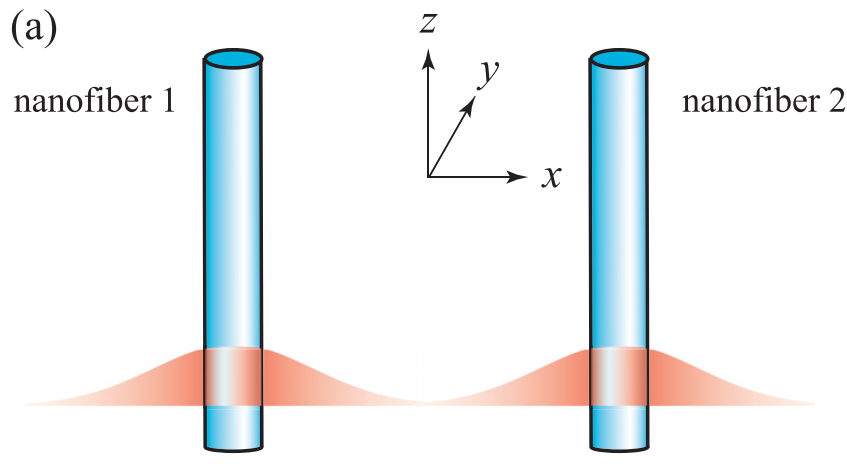

(b)

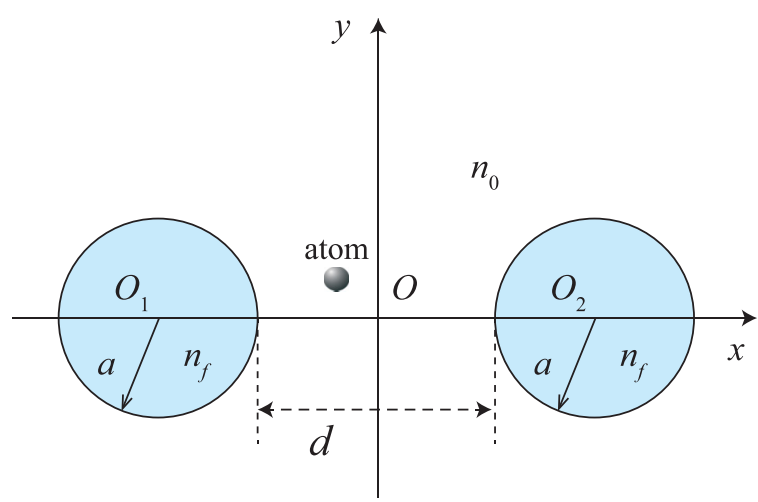

FIG. 1. Two coupled identical parallel optical nanofibers (a) and the geometry of the system (b).

between the nanofibers with a significant depth, a large scattering-limited coherence time, and a large trap lifetime.

The paper is organized as follows. In Sec. II we describe the model of two coupled identical parallel optical nanofibers and discuss the properties of the spatial field distribution in the odd $\mathcal{E}_{z}$-sine array mode. In Sec. III, we calculate the trapping potential of an atom outside the nanofibers. Finally, we conclude in Sec. IV.

\section{ODD $\mathcal{E}_{z}$-SINE ARRAY MODE OF TWO IDENTICAL PARALLEL NANOFIBERS}

We study two identical vacuum-clad, optical nanofibers that are aligned parallel to each other in the direction of the fiber axis $z$ (see Fig. 1). The fibers are labeled by the indices $j=1$ and 2. Each nanofiber $j$ can be treated as a dielectric cylinder with a radius of $a_{j}=a$ and a refractive index of $n_{j}=$ $n_{f}>1$, surrounded by an infinite background of vacuum or air with a refractive index of $n_{0}=1$. The nanofiber diameters are a few hundreds of nanometers. Depending on the fiber size parameter $V=k a \sqrt{n_{f}^{2}-n_{0}^{2}}$, an individual nanofiber $j$ can support either a single mode or multiple modes. Here, $k=\omega / c$ is the wave number of light with the optical frequency $\omega$ in free space. We are interested in the normal modes of the two-fiber system. We assume that the fibers are fixed in space and are, therefore, not interested in the van der Waals interaction between them.

We introduce the global Cartesian coordinate system $\{x, y, z\}$. Here, the $z$ axis is parallel to the $z_{1}$ and $z_{2}$ axes of the fibers, the $x$ axis is perpendicular to the $z$ axis and connects the centers $O_{1}$ and $O_{2}$ of the fibers, and the $y$ axis is perpendicular to the $x$ and $z$ axes (see Fig. 1). The plane $x y$ is the transverse (cross-sectional) plane of the fibers. The $x$ and $y$ axes are called the radial and tangential axes, respectively, of the two-fiber system [see Fig. 1(b)]. The positions of the fiber centers $O_{1}$ and $O_{2}$ on the $x$ axis are $O_{1}=-(a+d / 2)$ and $\mathrm{O}_{2}=a+d / 2$, where $d$ is the fiber separation distance. We introduce the polar coordinate system $\{r, \varphi\}$ associated with the central Cartesian coordinate system $\{x, y\}$. For each individual fiber $j$, we introduce the local polar coordinate system $\left\{r_{j}, \varphi_{j}\right\}$.

We consider a light field with an optical frequency, $\omega$, which propagates in the $+z$ direction with a propagation constant, $\beta$. The electric and magnetic components of the field can be written as $\mathbf{E}=\left[\mathcal{E} e^{-i(\omega t-\beta z)}+\right.$ c.c. $] / 2$ and $\mathbf{H}=$ $\left[\mathcal{H} e^{-i(\omega t-\beta z)}+\right.$ c.c. $] / 2$, respectively, where $\mathcal{E}$ and $\mathcal{H}$ are the slowly varying complex envelopes.

The normal modes of the coupled fibers are called array modes. The exact theory for the guided normal modes of two parallel dielectric cylinders has been formulated in Ref. [31]. According to Ref. [31], there are four kinds of normal modes, denoted as even $\mathcal{E}_{z}$-cosine, odd $\mathcal{E}_{z}$-cosine, even $\mathcal{E}_{z}$-sine, and odd $\mathcal{E}_{z}$-sine array modes. We are interested in the case where the fiber radii are small enough that no more than one normal mode of each of the four kinds can be supported. It has been shown that, for an odd $\mathcal{E}_{z}$-sine array mode of two coupled identical parallel fibers, the electric intensity distribution attains a local minimum of exactly zero at the two-fiber center (the midpoint between the nanofibers) [40].

We employ the theory of Ref. [31] to calculate the propagation constant and the spatial distribution of the field in an odd $\mathcal{E}_{z}$-sine array mode of two identical parallel vacuum-clad silica nanofibers [40]. The key results of Ref. [31] for this mode are summarized in the Appendix. We solve Eqs. (A6) and use the expressions (A1), (A2), and (A8) to calculate the components of the field. In our numerical calculations, the infinite number of circular harmonics is truncated at a finite number $N_{\max }$ in the range from 9 to 19. The value of $N_{\max }$ is chosen such that the propagation constant converges and the boundary conditions are satisfied with reasonable accuracy [31]. To calculate the refractive index $n_{f}$ of the silica nanofibers, we use the four-term Sellmeier formula for fused silica $[41,42]$.

We plot in Fig. 2 the propagation constant $\beta$ of the odd $\mathcal{E}_{z}$-sine array mode as functions of the fiber radius $a$, the light-field wavelength $\lambda$, and the fiber separation distance $d$. We observe from Figs. 2(a) and 2(b) that the odd $\mathcal{E}_{z}$-sine array mode has cutoffs in the dependencies of $\beta$ on $a$ and $\lambda$. The position of a cutoff is determined by the solution to the equation $\beta / k=1$ [31].

We display in Fig. 3 the cross-sectional profile and the axial profiles of the electric intensity distribution $|\mathcal{E}|^{2}$ of the field in the odd $\mathcal{E}_{z}$-sine array mode of two identical parallel nanofibers. We see from the figure that $|\mathcal{E}|^{2}$ is symmetric with respect to the principal axes $x$ and $y$. Figures 3(b) and 3(c) show that the electric field $\mathcal{E}$ of the odd $\mathcal{E}_{z}$-sine array mode is exactly equal to zero at the midpoint $(x, y)=(0,0)$ between the nanofibers. This feature of the odd $\mathcal{E}_{z}$-sine array mode can be used to trap ground-state atoms with a blue-detuned light field [43-45]. The existence of a local minimum of exactly zero at the two-fiber center is due to the destructive interference between the fields of the individual fibers in the 


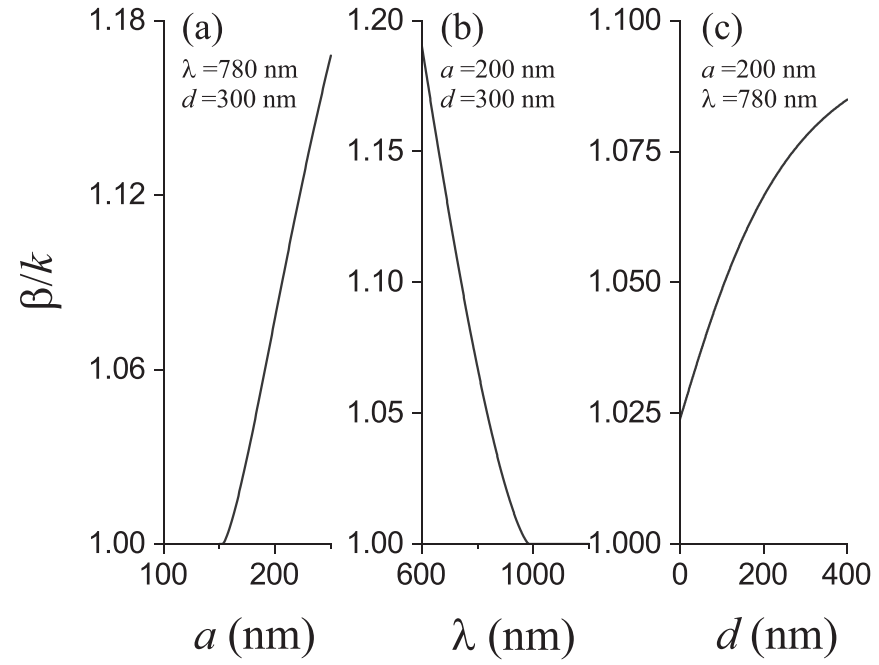

FIG. 2. The propagation constant $\beta$ of the odd $\mathcal{E}_{z}$-sine array mode, normalized to the free-space wave number $k$, as functions of (a) the fiber radius $a$, (b) the light-field wavelength $\lambda$, and (c) the fiber separation distance $d$. The parameters are (a) $\lambda=780 \mathrm{~nm}$ and $d=300 \mathrm{~nm}$, (b) $a=200 \mathrm{~nm}$ and $d=300 \mathrm{~nm}$, and (c) $a=200 \mathrm{~nm}$ and $\lambda=780 \mathrm{~nm}$. The refractive index of the fibers is $n_{f}=1.4537$ in panels (a) and (c) and is calculated from the four-term Sellmeier formula for fused silica $[41,42]$ in panel (b). The refractive index of the surrounding medium is $n_{0}=1$.

odd $\mathcal{E}_{z}$-sine array mode and occurs for any fiber separation distance $d$ [40]. The odd array modes of two coupled nanofibers behave like the dark states of a three-level atom driven by two light fields.

We emphasize that the local minimum of the electric intensity distribution is positioned at the midpoint between the nanofibers only in the case of identical nanofibers. According to Ref. [40], the intensity distributions of the guided array modes are sensitive to the difference between the radii of the nanofibers. A slight difference between the radii of the nanofibers may lead to a significant shift of the position of the local minimum of the optical potential toward the larger nanofiber. For example, for two nanofibers with the radii of 200 and $220 \mathrm{~nm}$, separated by a distance of $300 \mathrm{~nm}$, the local minimum of the optical potential is about $50 \mathrm{~nm}$ from the surface of the larger nanofiber. In this case, it is not easy to realize an optical trap that can beat the substantial effect of the van der Waals potential.

Due to the similar underlying physics, antisymmetric modes of slotted fibers $[20,21]$ and alligator photonic crystal waveguides [22-25] might support a similar trapping scheme. Furthermore, a Y-shape structure, that is, two fibers crossed at an acute angle, might also support a similar trap within a certain region. This might be an advantage for controlling the atom trapping possibility along the $z$ direction.

The destructive interference between the individual fiber modes in the odd $\mathcal{E}_{z}$-sine array mode plays an important role in producing a local minimum of the intensity distribution at the midpoint between the fibers with a tight confinement along the $x$ and $y$ axes. When we sum up the intensities of the quasicircularly polarized fundamental modes of two independent fibers, the midpoint between the fibers is a local minimum along the $x$ axis and a local maximum along the $y$
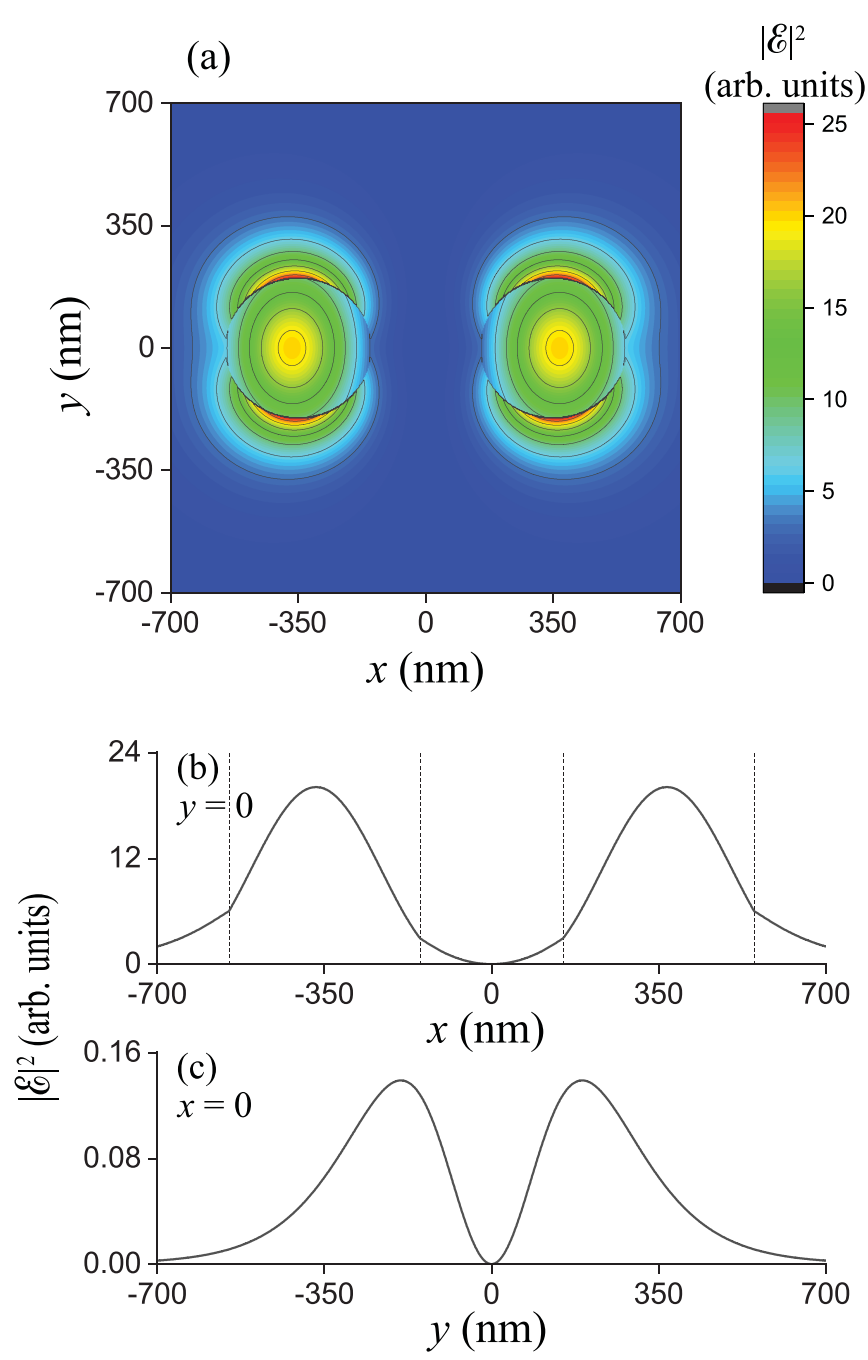

FIG. 3. (a) Cross-sectional profile and (b) $x$ - and (c) $y$-axial profiles of the electric intensity distribution $|\mathcal{E}|^{2}$ of the field in the odd $\mathcal{E}_{z}$-sine array mode of two identical parallel nanofibers. The vertical dashed lines in panel (b) indicate the positions of the fiber surfaces on the $x$ axis. The wavelength of light is $\lambda=780 \mathrm{~nm}$, the fiber radius is $a=200 \mathrm{~nm}$, and the separation distance between the fibers is $d=300 \mathrm{~nm}$. Other parameters are as in Fig. 2 .

axis, that is, a saddle point in the fiber transverse plane $(x, y)$. It is not easy to get a local minimum with a tight confinement along the $x$ and $y$ axes using two mutually incoherent fields.

\section{TRAPPING POTENTIAL OF AN ATOM OUTSIDE THE NANOFIBERS}

We consider an alkali-metal atom moving in the optical potential generated by an off-resonant guided light field in the odd $\mathcal{E}_{z}$-sine array mode outside the two nanofibers.

\section{A. Optical potential}

We assume that the atom is in the ground state and the field is off resonance with the atom. We ignore the field-induced coupling and mixing of the Zeeman sublevels of the atomic state. The optical potential $U_{\text {opt }}$ of the atom in the field is then 
given by [46]

$$
U_{\text {opt }}=-\frac{1}{4} \alpha|\mathcal{E}|^{2},
$$

where $\alpha=\alpha(\omega)$ is the real part of the scalar dynamical polarizability of the atom at the optical frequency $\omega$. The factor $1 / 4$ in Eq. (1) results from the fact that the dipole of the atom is not a permanent dipole but is induced by the field, giving $1 / 2$, and from the fact that the intensity is averaged over optical oscillations, giving another $1 / 2$.

The function $\alpha(\omega)$ for a ground-state alkali-metal atom is given by [46]

$$
\alpha(\omega)=2 \pi \epsilon_{0} c^{3} \sum_{j} \frac{g_{j}}{g_{a}} \frac{A_{j a}\left(1-\omega^{2} / \omega_{j a}^{2}\right)}{\left(\omega_{j a}^{2}-\omega^{2}\right)^{2}+\gamma_{j}^{2} \omega^{2}} .
$$

Here, $g_{j}$ and $g_{a}$ are the statistical weights of the excited level $|j\rangle$ and the ground-state level $|a\rangle$, respectively, $\omega_{j a}$ and $A_{j a}$ are the frequency and emission transition probability, respectively, of the spectral line $j a$, and $\gamma_{j}$ is the lifetime of the excited level $|j\rangle$. We note that the vector polarizability is neglected in Eqs. (1) and (2) and the tensor polarizability vanishes for the ground-state alkali-metal atom [47,48].

To be specific, we consider atomic cesium. A ground-state cesium atom has two strong transitions, at $852 \mathrm{~nm}\left(D_{2}\right.$ line $)$ and $894 \mathrm{~nm}\left(D_{1}\right.$ line). In order to trap the atom, we use the wavelength $\lambda=780 \mathrm{~nm}$, which is blue-detuned from the $D_{1}$ and $D_{2}$ lines. The dynamical polarizability of the ground-state cesium atom has been calculated numerically $[6,47,48]$. To make simple calculations for the dynamical polarizability, we follow Ref. [6] and take into account the four most dominant lines of the atom, namely, $\lambda_{1 a}=852.113 \mathrm{~nm}, \lambda_{2 a}=$ $894.347 \mathrm{~nm}, \lambda_{3 a}=455.528 \mathrm{~nm}$, and $\lambda_{4 a}=459.317 \mathrm{~nm}$ (see Ref. [49]). The emission transition probabilities of these four lines are $A_{1 a}=3.276 \times 10^{7} \mathrm{~s}^{-1}, A_{2 a}=2.87 \times 10^{7} \mathrm{~s}^{-1}$, $A_{3 a}=1.88 \times 10^{6} \mathrm{~s}^{-1}$, and $A_{4 a}=8 \times 10^{5} \mathrm{~s}^{-1}$. The statistical weights of the upper states are $g_{1}=4, g_{2}=2, g_{3}=4$, and $g_{4}=2$, and that of the ground state is $g_{a}=2$. For the wavelength $\lambda=780 \mathrm{~nm}$, the polarizability of the atom is estimated to be $\alpha \cong-1709$ a.u., which is negative and hence gives the repulsive optical potential $U_{\text {opt }}=(1 / 4)|\alpha||\mathcal{E}|^{2}$.

The power of the guided light is given by $P=$ $(1 / 2) \int \operatorname{Re}\left[\mathcal{E} \times \mathcal{H}^{*}\right]_{z} d \mathbf{r}$, where $\mathbf{r}=(x, y)$ and $\int d \mathbf{r}=$ $\int_{-\infty}^{\infty} d x \int_{-\infty}^{\infty} d y$. It is clear that the magnitude and the depth of the optical potential $U_{\text {opt }}$ increase linearly with increasing electric field intensity $|\mathcal{E}|^{2}$ and, hence, with increasing power of light $P$.

We plot in Fig. 4 the spatial profile of the optical potential $U_{\text {opt }}$ of a ground-state cesium atom in the area $\left\{r_{1}, r_{2}>\right.$ $a ;|x|<a+d / 2 ;|y|<a\}$, which lies between the two fibers. The inset of the figure shows $U_{\text {opt }}$ in a small vicinity of the two-fiber center $O=(0,0)$. We observe that $U_{\text {opt }}$ has a local minimum of exactly zero at $O$. This feature can be used to trap atoms. Due to the geometry of the system, the optical potential is not cylindrically symmetric.

We note that the power of light $P=100 \mathrm{~mW}$ is used for the calculations of the optical potential $U_{\text {opt }}$ in Fig. 4. This power for the two-fiber system means that each of the two fibers experiences an effective power of $50 \mathrm{~mW}$, twice the value of $25 \mathrm{~mW}$ used in the experiment on trapping of atoms with a single nanofiber [7]. Dropping the power by a

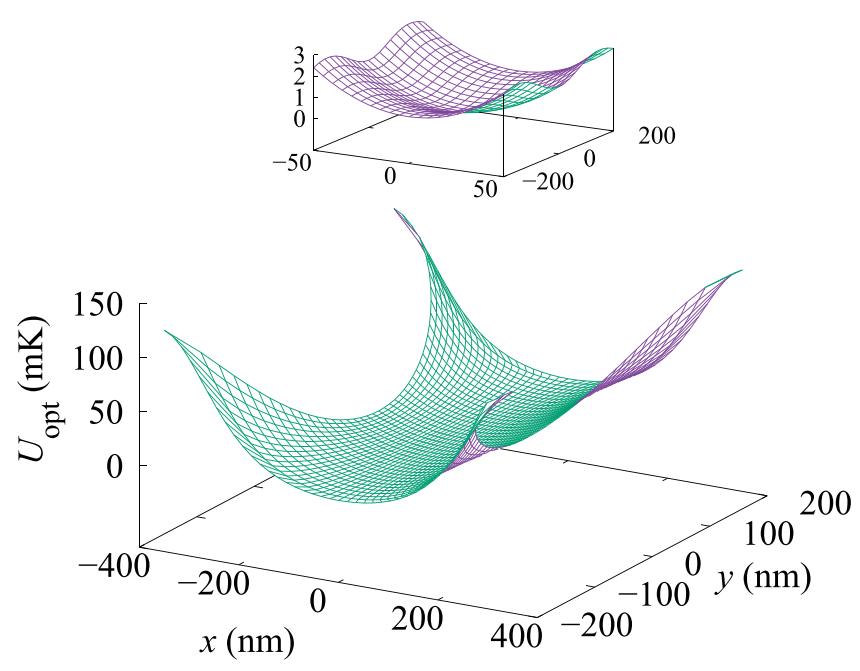

FIG. 4. Spatial profile of the optical potential $U_{\text {opt }}$ of a groundstate cesium atom in the area $\left\{r_{1}, r_{2}>a ;|x|<a+d / 2 ;|y|<a\right\}$, which lies between the two fibers. The inset shows $U_{\text {opt }}$ in a small vicinity of the two-fiber center $O=(0,0)$. The trapping field is in the odd $\mathcal{E}_{z}$-sine array mode with the power of $100 \mathrm{~mW}$. Other parameters are as in Fig. 3.

factor of 2 would reduce the magnitude and the depth of the total trapping potential by almost the same factor (see Fig. 7 in Sec. IIID). It is worth mentioning here the availability of 250-nm-radius ultrahigh-transmission nanofibers that can withstand the application of $400 \mathrm{~mW}$ from a laser at $760 \mathrm{~nm}$ under high-vacuum conditions [50].

The rate of spontaneous scattering caused by a light field $\mathcal{E}$ is given by

$$
\Gamma_{\mathrm{sc}}=\frac{1}{4 \hbar} \kappa|\mathcal{E}|^{2},
$$

where $\kappa=\kappa(\omega)$ is the imaginary part of the scalar dynamical polarizability of the atom. The function $\kappa(\omega)$ for a groundstate alkali-metal atom is given by [46]

$$
\kappa(\omega)=2 \pi \epsilon_{0} c^{3} \sum_{j} \frac{g_{j}}{g_{a}} \frac{A_{j a} \gamma_{j a} \omega / \omega_{j a}^{2}}{\left(\omega_{j a}^{2}-\omega^{2}\right)^{2}+\gamma_{j}^{2} \omega^{2}} .
$$

For atoms spending time in a motional quantum state $|\psi\rangle$, the average scattering rate is $\left\langle\Gamma_{\mathrm{sc}}\right\rangle \equiv\left\langle\psi\left|\Gamma_{\mathrm{sc}}\right| \psi\right\rangle=$ $(\kappa / 4 \hbar)\left\langle|\mathcal{E}|^{2}\right\rangle$, with $\left\langle|\mathcal{E}|^{2}\right\rangle \equiv\left\langle\psi\left|\left(|\mathcal{E}|^{2}\right)\right| \psi\right\rangle$. The characteristic scattering-limited coherence time of the trap is

$$
\tau_{\mathrm{coh}}=\frac{1}{\left\langle\Gamma_{\mathrm{sc}}\right\rangle} .
$$

A scattered photon imparts a recoil energy of $E_{\text {rec }}=$ $(\hbar k)^{2} / 2 M$ to the atom, where $M$ is the mass of the atom. Therefore, the absorption of incident photons and the emission of other photons result in a loss of atoms from the trapping potential. For a trap depth $U_{D}$, the trap lifetime due to recoil heating is given by [51]

$$
\tau_{\text {trap }}=\frac{U_{D}}{2 E_{\mathrm{rec}}\left\langle\Gamma_{\mathrm{sc}}\right\rangle} \text {. }
$$

When the light-field frequency is far from the atomic resonances and the field at the trapping potential minimum is 
weak, the scattering rate is small and, therefore, the scatteringlimited coherence time and the trap lifetime are large.

\section{B. van der Waals potential}

An atom near the surface of a medium undergoes a van der Waals force. The van der Waals potential of an atom at a radial position $r$ near the surface of a cylindrical dielectric rod of radius $a$, with $r>a$, is given by [52]

$$
\begin{aligned}
V(r)= & \frac{\hbar}{4 \pi^{3} \epsilon_{0}} \sum_{n=-\infty}^{\infty} \int_{0}^{\infty} d \beta\left[\beta^{2} K_{n}^{\prime 2}(\beta r)\right. \\
& \left.+\left(\beta^{2}+n^{2} / r^{2}\right) K_{n}^{2}(\beta r)\right] \int_{0}^{\infty} d \xi \alpha(i \xi) G_{n}(i \xi, \beta),
\end{aligned}
$$

where

$$
G_{n}(i \xi, \beta)=\frac{\left[\epsilon(i \xi)-\epsilon_{0}\right] I_{n}(\beta a) I_{n}^{\prime}(\beta a)}{\epsilon_{0} I_{n}(\beta a) K_{n}^{\prime}(\beta a)-\epsilon(i \xi) I_{n}^{\prime}(\beta a) K_{n}(\beta a)} .
$$

Here $\epsilon(i \xi)$ is the dynamical dielectric function of the medium for the imaginary frequency $i \xi$, and $I_{n}$ and $K_{n}$ are the modified Bessel functions of the first and second kinds, respectively. The van der Waals potential of a ground-state cesium atom near a silica fiber has been calculated numerically [6]. Note that a different study, using the electrostatic approximation and the image-charge formalism, has been developed for the van der Waals interaction between an atom and the convex surface of a nanocylinder [53].

For an atom near two identical parallel optical nanofibers, the van der Waals potential is given as

$$
U_{\mathrm{vdW}}=V\left(r_{1}\right)+V\left(r_{2}\right) .
$$

Here, $r_{1}$ and $r_{2}$ are the distances from the atom to the fiber axes $z_{1}$ and $z_{2}$, respectively.

\section{Total potential}

The total potential of the atom is given as

$$
U=U_{\mathrm{opt}}+U_{\mathrm{vdW}} .
$$

We plot in Fig. 5(a) the cross-sectional profile of the total potential $U$ of a ground-state cesium atom in the vicinity of the two-fiber center $O=(0,0)$. In Figs. 5(b) and 5(c), we depict the axial profiles of the total potential $U$ in the region between the nanofibers. We observe from the figures that $U(x, y)$ has a negative local minimum $U_{\min } \equiv U(0,0)=$ $U_{\mathrm{vdW}}(0,0)<0$ at $O$. Comparison between the solid red lines and the dashed blue lines of Figs. 5(b) and 5(c) shows that the total potential $U$ is mainly determined by the optical potential $U_{\mathrm{opt}}$ in the vicinity of the two-fiber center $O$, which is positioned at a distance of $150 \mathrm{~nm}$ from the fiber surfaces in the case of the figures. This feature is a consequence of the fact that the effects of the van der Waals potential on the total trapping potential around the minimum point are not significant when the fiber separation distance and the power of the guided light field are large enough. Like the optical potential $U_{\text {opt }}$, the total potential $U$ is not cylindrically symmetric due to the geometry of the system. Figures 5(b) and 5(c) and the scales of their vertical axes show that the depth of the profile
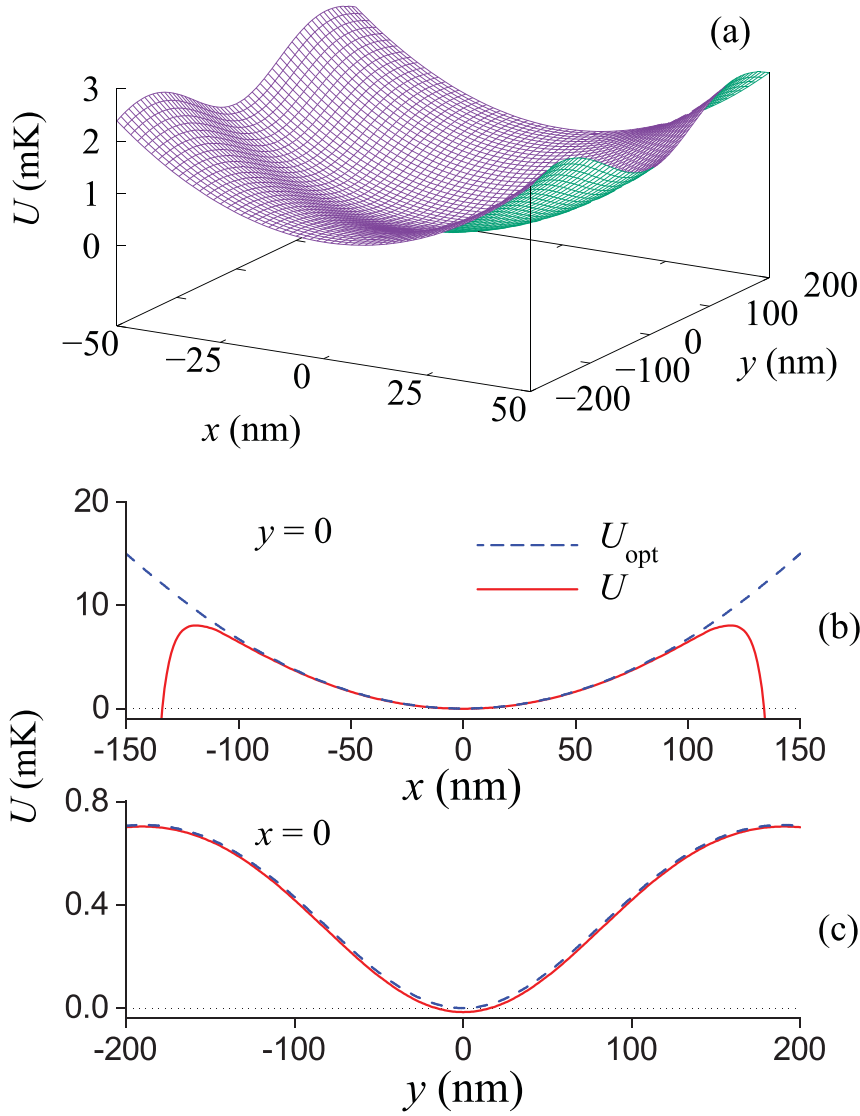

FIG. 5. (a) Cross-sectional profile of the total potential $U$ of a ground-state cesium atom in a small area around the two-fiber center $O=(0,0)$. (b) and (c) Axial profiles of the total potential $U$ (solid red lines) and the optical potential $U_{\text {opt }}$ (dashes blue lines) of a ground-state cesium atom along the $x$ and $y$ axes. Parameters used are as in Figs. 3 and 4.

of the potential along the $x$ axis is larger than that of the profile of the potential along the $y$ axis. Therefore, the depth of the potential profile along the $y$ axis is the effective depth of the trapping potential.

The one-dimensional motion of the atom along the $x$ or $y$ axis can be treated as the motion of a particle in the potential $U_{x}(x)=U(x, 0)$ or $U_{y}(y)=U(0, y)$. In Fig. 6, we plot the wave functions of the first five levels of the onedimensional motion of the atom in the potentials $U_{x}$ and $U_{y}$ for the case of Fig. 5. We find that the trap frequencies are $\omega_{x} / 2 \pi \cong 1441 \mathrm{kHz}$ and $\omega_{y} / 2 \pi \cong 438 \mathrm{kHz}$. The spacing between the the energies of the ground state and the first excited state is roughly equal to $\hbar \omega_{x} \cong k_{B} \times 69 \mu \mathrm{K}$ for the motion along the $x$ axis and $\hbar \omega_{y} \cong k_{B} \times 21 \mu \mathrm{K}$ for the motion along the $y$ axis. The characteristic sizes of the corresponding motional ground states are $\Delta x=\sqrt{\hbar / 2 M \omega_{x}} \cong 5.1 \mathrm{~nm}$ and $\Delta y=\sqrt{\hbar / 2 M \omega_{y}} \cong 9.3 \mathrm{~nm}$. The trap frequencies and the motional ground-state sizes characterize the tightness of the trap. The values obtained above are comparable to the corresponding characteristic values for the single-nanofiber two-color traps $[7,8,10-13]$.

The scattering rates for the ground states of the atomic motions along the $x$ and $y$ axes in the case of Fig. 5 are 


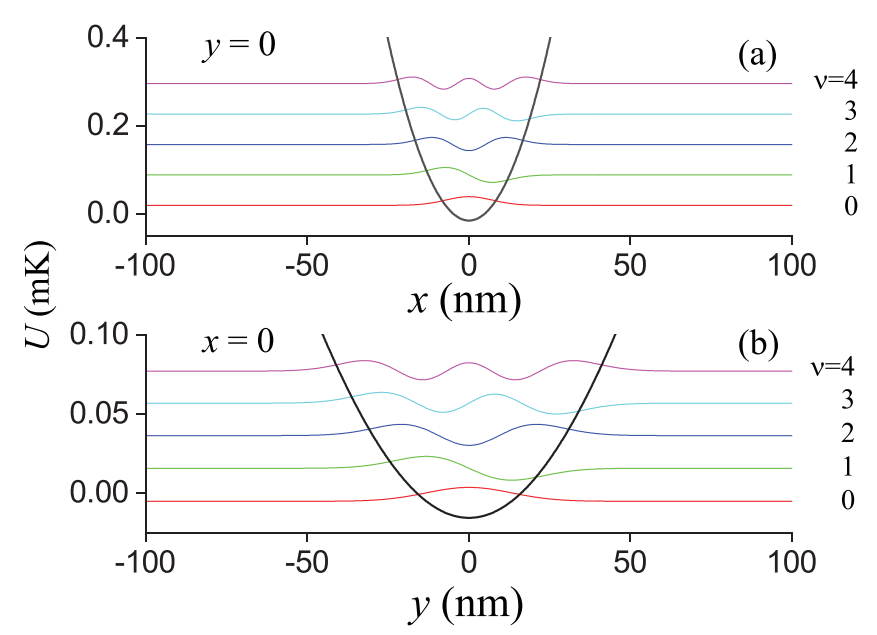

FIG. 6. Bound states for the first five levels $(v=0,1,2,3$, and 4 ) of the one-dimensional motion of a ground-state cesium atom in the potential $U$ along the $x$ and $y$ axes. Parameters used are as in Figs. 3-5.

found to be $\left\langle\Gamma_{\text {sc }}\right\rangle_{x} \cong 0.17 \mathrm{~s}^{-1}$ and $\left\langle\Gamma_{\text {sc }}\right\rangle_{y} \cong 0.05 \mathrm{~s}^{-1}$. The scattering rate $\left\langle\Gamma_{\mathrm{sc}}\right\rangle$ of the atom in the trap is estimated as the maximal value of $\left\langle\Gamma_{\mathrm{sc}}\right\rangle_{x}$ and $\left\langle\Gamma_{\mathrm{sc}}\right\rangle_{y}$, and is, in the case of Fig. 5, given as $\left\langle\Gamma_{\text {sc }}\right\rangle \cong 0.17 \mathrm{~s}^{-1}$. The corresponding scattering-limited coherence time is $\tau_{\text {coh }} \equiv 1 /\left\langle\Gamma_{\text {sc }}\right\rangle \cong 5.8 \mathrm{~s}$. The trap depth is estimated as the minimal value of the trap depths for the motions along the $x$ and $y$ axes and is, in the case of Fig. 5(c), equal to $U_{D} \cong 0.7 \mathrm{mK}$, comparable to the depths of the single-nanofiber two-color traps [7,8,10-13]. The corresponding recoil-limited trap lifetime is $\tau_{\text {trap }} \cong 4.8 \mathrm{~h}$. This value is much larger than the recoil-limited lifetimes of 100 and 30 s estimated for the single-nanofiber two-color traps in the experiments in Refs. [7,8], respectively.

Our trapping scheme deals with only the motion of the atom in the two-dimensional fiber cross-sectional plane. In this scheme, the atom can move freely in the fiber axis direction $z$. Consequently, the scheme can be referred to as a waveguide for atoms.

\section{Dependencies of the trapping potential on the parameters of the fibers and the light field}

The trapping potential $U$ depends on the light-field power $P$, the fiber radius $a$, the light-field wavelength $\lambda$, and the fiber separation distance $d$.

We plot in Fig. 7 the trapping potential $U$ for different values of the power $P$ of the guided light. It is clear from the figure that the magnitude and the depth of the trapping potential are almost linearly proportional to the power. This feature is a result of the fact that, when the position $\mathbf{r}$ is not too close to the fiber surfaces, the total potential $U(\mathbf{r})$ is mainly determined by the optical potential $U_{\text {opt }}(\mathbf{r})$, which is linearly proportional to the power of light [see Eq. (1)].

We plot in Fig. 8 the trapping potential $U$ for different values of the fiber radius $a$. We observe from the figure that, when $a$ is small (see the dotted red lines) or large (see the dashed green lines), $U$ is shallow. These features are consequences of

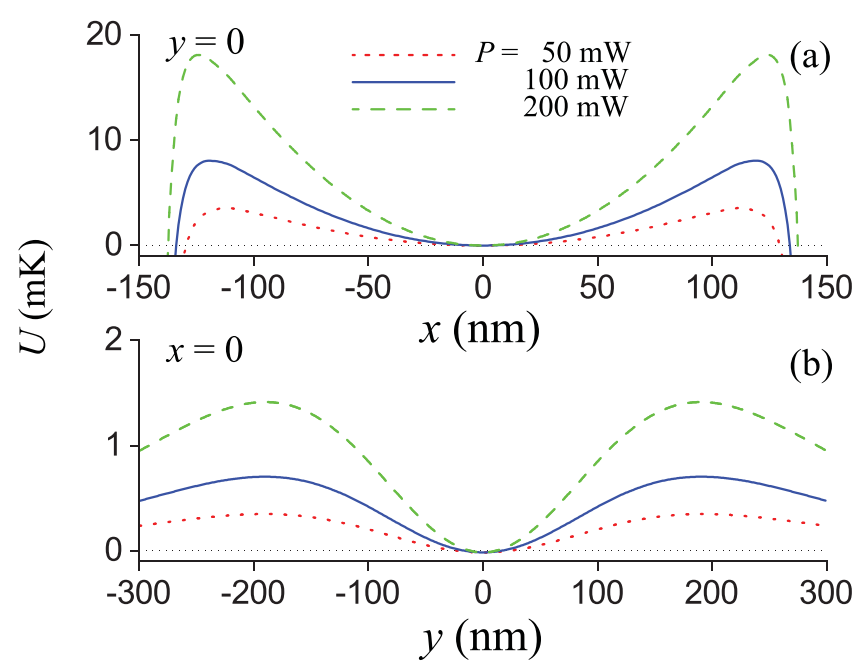

FIG. 7. Axial profiles of the total potential $U$ of a ground-state cesium atom for different values $P=50 \mathrm{~mW}$ (dotted red lines), 100 $\mathrm{mW}$ (solid blue lines), and $200 \mathrm{~mW}$ (dashed green lines) of the lightfield power. The fiber radius is $a=200 \mathrm{~nm}$, the wavelength of light is $\lambda=780 \mathrm{~nm}$, and the fiber separation distance is $d=300 \mathrm{~nm}$. Other parameters are as in Figs. 3-5.

the wide spread of the guided field outside a thin fiber and the tight confinement of the guided field inside a thick fiber.

We plot in Fig. 9 the trapping potential $U$ for different values of the wavelength of light $\lambda$. The figure shows that, when $\lambda$ is closer to the resonance with the atom $(852 \mathrm{~nm}$ for the atomic cesium $D_{2}$ line), $U$ is deeper. These features appear as consequences of the dependence of the atomic polarizability on the wavelength of a detuned light field. Note that the dependence of the trapping potential on the wavelength of light occurs through not only the atomic polarizability but

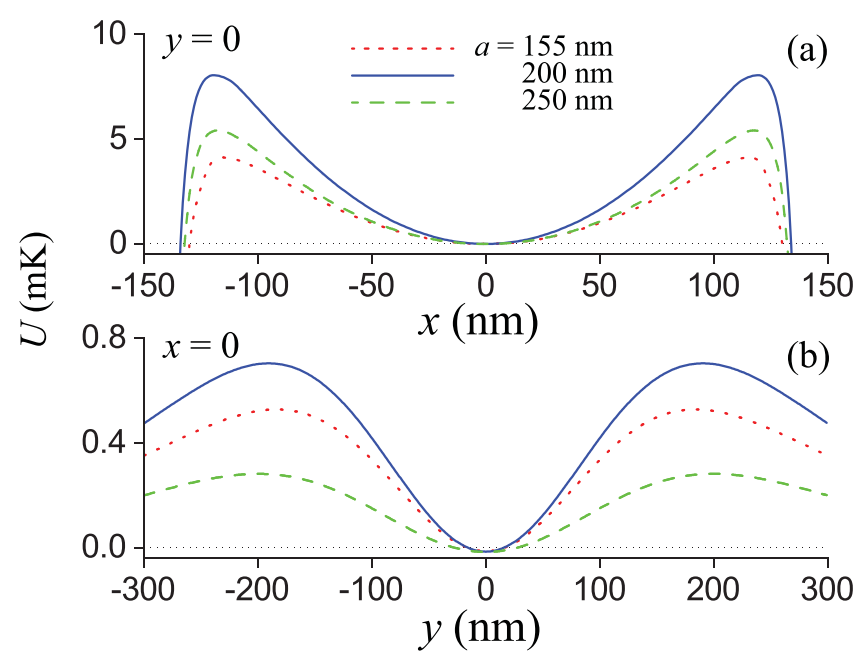

FIG. 8. Axial profiles of the total potential $U$ of a ground-state cesium atom for different values $a=155 \mathrm{~nm}$ (dotted red lines), $200 \mathrm{~nm}$ (solid blue lines), and $250 \mathrm{~nm}$ (dashed green lines) of the fiber radius. The wavelength of light is $\lambda=780 \mathrm{~nm}$, the separation distance between the fibers is $d=300 \mathrm{~nm}$, and the power of light is $P=100 \mathrm{~mW}$. Other parameters are as in Figs. $3-5$. 


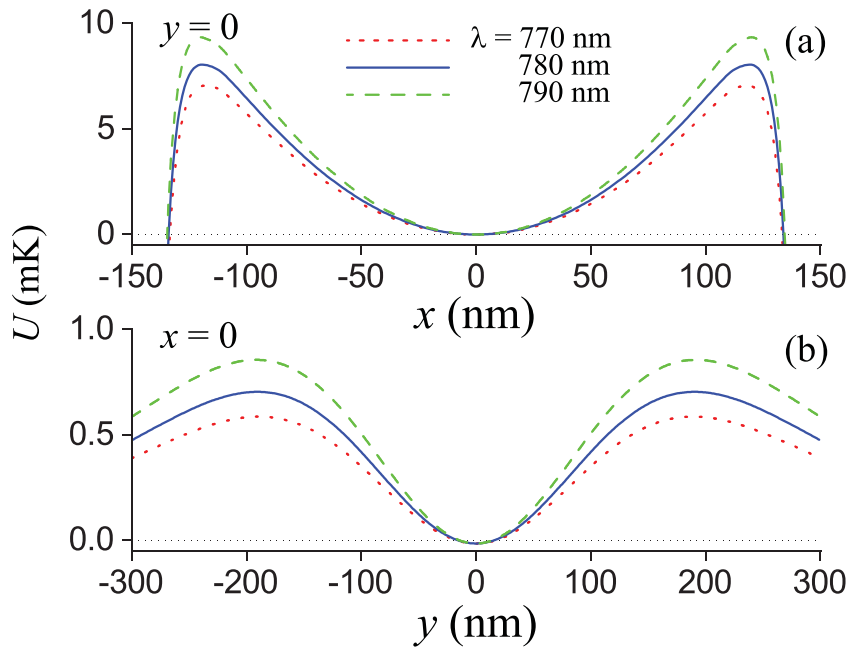

FIG. 9. Axial profiles of the total potential $U$ of a ground-state cesium atom for different values $\lambda=770 \mathrm{~nm}$ (dotted red lines), $780 \mathrm{~nm}$ (solid blue lines), and $790 \mathrm{~nm}$ (dashed green lines) of the wavelength of light. The fiber radius is $a=200 \mathrm{~nm}$, the separation distance between the fibers is $d=300 \mathrm{~nm}$, and the power of light is $P=100 \mathrm{~mW}$. Other parameters are as in Figs. 3-5.

also the mode profile and, consequently, the field intensity distribution.

We plot in Fig. 10 the trapping potential $U$ for different values of the fiber separation distance $d$. The figure shows that an increase in the separation distance $d$ leads to an increase in the depth of the trap along the $x$ direction [see Fig. 10(a)] and to a decrease in the depth of the trap along the $y$ direction [see Fig. 10(b)]. We also observe that an increase in $d$ leads to an almost linear increase in the width of the trapping potential along the $x$ axis but does not affect much the width of the trapping potential along the $y$ axis.

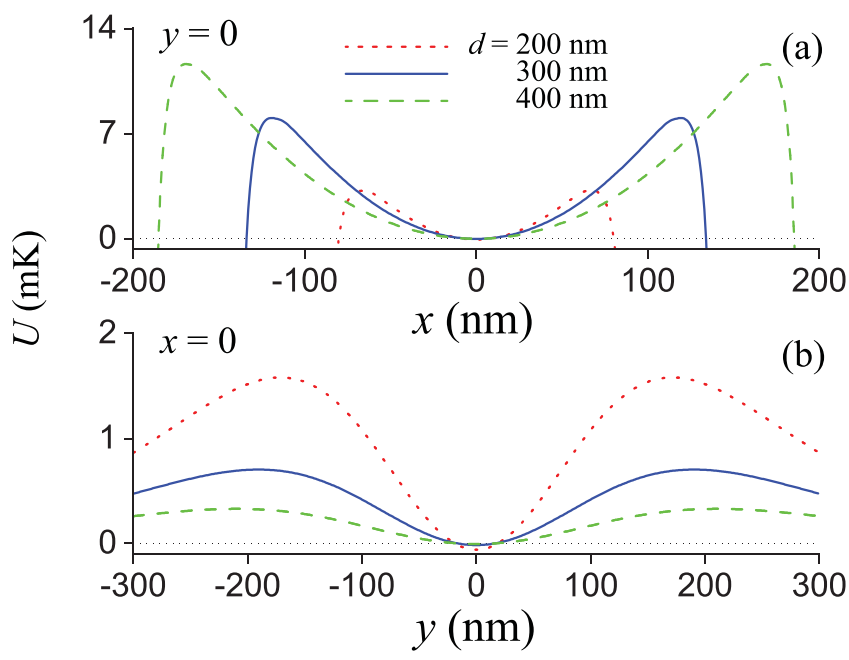

FIG. 10. Axial profiles of the total potential $U$ of a ground-state cesium atom for different values $d=200 \mathrm{~nm}$ (dotted red lines), $300 \mathrm{~nm}$ (solid blue lines), and $400 \mathrm{~nm}$ (dashed green lines) of the separation distance between the fibers. The fiber radius is $a=200 \mathrm{~nm}$, the wavelength of light is $\lambda=780 \mathrm{~nm}$, and the power of light is $P=100 \mathrm{~mW}$. Other parameters are as in Figs. 3-5.
When the fiber separation distance $d$ increases, the overlap and, hence, the interference between the modes of the individual fibers reduce. Since the interference is destructive in the case of the odd $\mathcal{E}_{z}$-sine array mode, an increase of the distance $d$ leads to an increase of the intensity of the array-mode field in the proximity of the fiber surfaces and, hence, to an increase of the trap depth along the $x$ axis [see Fig. 10(a)]. With the help of additional calculations not presented in this paper, we can show that the trap depth along the $x$ axis saturates when $d$ is large enough.

\section{SUMMARY}

In this work, we have studied the trapping potential of a ground-state cesium atom in a small region around the midpoint between two coupled identical parallel optical nanofibers. We have suggested to use a blue-detuned guided light field in the odd $\mathcal{E}_{z}$-sine array mode of the two nanofibers to produce an optical potential with a local minimum at the midpoint between the nanofibers. The vanishing of the field at the two-fiber center is a result of the destructive interference between the coupled modes of the individual nanofibers. We have demonstrated that the effects of the van der Waals potential on the total trapping potential around the minimum point are not significant when the fiber separation distance and the power of the guided light field are large. We have shown that, for appropriate realistic parameters, a net potential with a significant depth, a large scattering-limited coherence time, and a large trap lifetime can be obtained. We have demonstrated, for example, that a pair of 200-nm-radius silica fibers carrying $100 \mathrm{~mW}$ of 780-nm-wavelength light in the odd $\mathcal{E}_{z}$-sine array mode gives for cesium atoms a trap depth of $0.7 \mathrm{mK}$, a scattering-limited coherence time of $5.8 \mathrm{~s}$, and a recoil-heating-limited trap lifetime of $4.8 \mathrm{~h}$. Due to the geometry of the system, the trapping potential is not cylindrically symmetric. The depth of the trapping potential profile along the tangential direction $y$ is smaller than that of the trapping potential profile along the radial direction $x$ and is, hence, the effective trap depth. The dependencies of the trapping potential on the power of the guided light field, the fiber radius, the wavelength of light, and the fiber separation distance have been investigated. It has been shown that, at a position that is not too close to the fiber surfaces, the total potential is mainly determined by the optical potential and, hence, is linearly proportional to the power of light. When the fiber radius is small or large enough, the potential at the midpoint between the nanofibers is shallow due to the wide spread of the guided field outside a thin fiber and the tight confinement of the guided field inside a thick fiber. We have observed that the wavelength of light affects the trapping potential through the atomic polarizability and the field intensity distribution. We have found that the depth of the trapping potential decreases with increasing fiber separation distance.

The aforementioned results have been obtained for the case where the nanofibers are identical to each other. Our additional calculations show that a slight difference on the order of $10 \%$ between the radii of the nanofibers may lead to a significant shift of the position of the local minimum of the optical potential toward the larger nanofiber. In this case, 
an optical trap may not work because of the substantial effect of the van der Waals potential.

\section{ACKNOWLEDGMENTS}

This work was supported by the Okinawa Institute of Science and Technology Graduate University and by the JSPS Grant-in-Aid for Scientific Research (C) 20K03795. The authors would like to acknowledge useful suggestions by an anonymous reviewer in relation to an alternative nanofiberbased trap configuration.

\section{APPENDIX: ODD $\mathcal{E}_{z}$-SINE ARRAY MODE}

According to Ref. [31], for an odd $\mathcal{E}_{z}$-sine guided normal (array) mode, the longitudinal components $\mathcal{E}_{z}$ and $\mathcal{H}_{z}$ of the electric and magnetic parts, respectively, of the field are given, inside fiber $j=1$ and 2 , as

$$
\begin{aligned}
\mathcal{E}_{z} & =\sum_{n=0}^{\infty} E_{n j} J_{n}\left(h r_{j}\right) \sin n \varphi_{j}, \\
\mathcal{H}_{z} & =\sum_{n=0}^{\infty} F_{n j} J_{n}\left(h r_{j}\right) \cos n \varphi_{j},
\end{aligned}
$$

and, outside the two fibers, as

$$
\begin{aligned}
\mathcal{E}_{z} & =\sum_{j=1}^{2} \sum_{n=0}^{\infty} G_{n j} K_{n}\left(q r_{j}\right) \sin n \varphi_{j}, \\
\mathcal{H}_{z} & =\sum_{j=1}^{2} \sum_{n=0}^{\infty} H_{n j} K_{n}\left(q r_{j}\right) \cos n \varphi_{j} .
\end{aligned}
$$

Here, we have introduced the fiber parameters

$$
h=\sqrt{k^{2} n_{f}^{2}-\beta^{2}}, \quad q=\sqrt{\beta^{2}-k^{2} n_{0}^{2}},
$$

which determine the scales of the spatial variations of the field both inside and outside the fibers. In Eqs. (A1) and (A2), $E_{n j}, F_{n j}, G_{n j}$, and $H_{n j}$ are the mode expansion coefficients, $J_{n}$ represents the Bessel functions of the first kind, and $K_{n}$ represents the modified Bessel functions of the second kind.

The symmetry relations for the coefficients $E_{m j}, F_{m j}, G_{m j}$, and $H_{m j}$, with $j=1$ and 2 , are [31]

$$
\begin{array}{ll}
E_{m 2}=(-1)^{m} E_{m 1}, & F_{m 2}=(-1)^{m} F_{m 1}, \\
G_{m 2}=(-1)^{m} G_{m 1}, & H_{m 2}=(-1)^{m} H_{m 1} .
\end{array}
$$

The coefficients $E_{n 1}$ and $F_{n 1}$ are given by the following equations [31]:

$$
\begin{aligned}
& J_{n}(u) E_{n 1}=K_{n}(w) G_{n 1}+I_{n}(w) \sum_{m=0}^{\infty} g_{n m} G_{m 1}, \\
& J_{n}(u) F_{n 1}=K_{n}(w) H_{n 1}+I_{n}(w) \sum_{m=0}^{\infty} f_{n m} H_{m 1} .
\end{aligned}
$$

The coefficients $G_{n 1}$ and $H_{n 1}$ are nonzero solutions of the following equations [31]:

$$
\begin{aligned}
n\left(\frac{1}{u^{2}}+\frac{1}{w^{2}}\right)\left[K_{n}(w) G_{n 1}+I_{n}(w) \sum_{m=0}^{\infty} g_{n m} G_{m 1}\right] \\
-\frac{\omega \mu_{0}}{\beta}\left[\frac{J_{n}^{\prime}(u)}{u J_{n}(u)}+\frac{K_{n}^{\prime}(w)}{w K_{n}(w)}\right] K_{n}(w) H_{n 1} \\
-\frac{\omega \mu_{0}}{\beta}\left[\frac{J_{n}^{\prime}(u)}{u J_{n}(u)}+\frac{I_{n}^{\prime}(w)}{w I_{n}(w)}\right] I_{n}(w) \sum_{m=0}^{\infty} f_{n m} H_{m 1}=0 \\
\quad n\left(\frac{1}{u^{2}}+\frac{1}{w^{2}}\right)\left[K_{n}(w) H_{n 1}+I_{n}(w) \sum_{m=0}^{\infty} f_{n m} H_{m 1}\right] \\
-\frac{\omega \epsilon_{0}}{\beta}\left[\frac{n_{f}^{2} J_{n}^{\prime}(u)}{u J_{n}(u)}+\frac{n_{0}^{2} K_{n}^{\prime}(w)}{w K_{n}(w)}\right] K_{n}(w) G_{n 1} \\
-\frac{\omega \epsilon_{0}}{\beta}\left[\frac{n_{f}^{2} J_{n}^{\prime}(u)}{u J_{n}(u)}+\frac{n_{0}^{2} I_{n}^{\prime}(w)}{w I_{n}(w)}\right] I_{n}(w) \sum_{m=0}^{\infty} g_{n m} G_{m 1}=0
\end{aligned}
$$

Here, we have introduced the coefficients

$$
\begin{aligned}
f_{n m} & =K_{m+n}(q W)+K_{m-n}(q W) \text { for } n>0, \\
f_{0, m} & =K_{m}(q W), \\
g_{n m} & =-K_{m+n}(q W)+K_{m-n}(q W),
\end{aligned}
$$

where $W=d+a_{1}+a_{2}$ is the distance between the fiber centers.

In terms of the longitudinal components $\mathcal{E}_{z}$ and $\mathcal{H}_{z}$ of the field, the transverse components $\mathcal{E}_{x, y}$ and $\mathcal{H}_{x, y}$ are given as [26-28]

$$
\begin{aligned}
\mathcal{E}_{x} & =\frac{i \beta}{k^{2} n_{\mathrm{ref}}^{2}-\beta^{2}}\left(\frac{\partial}{\partial x} \mathcal{E}_{z}+\frac{\omega \mu_{0}}{\beta} \frac{\partial}{\partial y} \mathcal{H}_{z}\right), \\
\mathcal{E}_{y} & =\frac{i \beta}{k^{2} n_{\mathrm{ref}}^{2}-\beta^{2}}\left(\frac{\partial}{\partial y} \mathcal{E}_{z}-\frac{\omega \mu_{0}}{\beta} \frac{\partial}{\partial x} \mathcal{H}_{z}\right), \\
\mathcal{H}_{x} & =\frac{i \beta}{k^{2} n_{\mathrm{ref}}^{2}-\beta^{2}}\left(\frac{\partial}{\partial x} \mathcal{H}_{z}-\frac{\omega \epsilon_{0} n_{\mathrm{ref}}^{2}}{\beta} \frac{\partial}{\partial y} \mathcal{E}_{z}\right), \\
\mathcal{H}_{y} & =\frac{i \beta}{k^{2} n_{\mathrm{ref}}^{2}-\beta^{2}}\left(\frac{\partial}{\partial y} \mathcal{H}_{z}+\frac{\omega \epsilon_{0} n_{\mathrm{ref}}^{2}}{\beta} \frac{\partial}{\partial x} \mathcal{E}_{z}\right) .
\end{aligned}
$$

Here, $n_{\text {ref }}$ is the spatial distribution of the refractive index of the two-fiber system, that is, $n_{\text {ref }}=n_{f}$ inside the fibers and $n_{\text {ref }}=n_{0}$ outside the two fibers.

The dispersion equation for the propagation constant of the mode is $\Delta=0$, where $\Delta$ is the determinant of the system of linear equations (A6) for $G_{n j}$ and $H_{n j}$. The solution to the equation $\Delta=0$ determines the propagation constant $\beta$, which allows us to calculate the other fiber parameters $h$ and $q$ [see Eqs. (A3)].

We make $E_{n j}, F_{n j}, G_{n j}$, and $H_{n j}$ real-valued coefficients by omitting a common global phase. Then, for the electric part of the field, we have $\mathcal{E}_{z}^{*}=\mathcal{E}_{z}, \mathcal{E}_{x}^{*}=-\mathcal{E}_{x}$, and $\mathcal{E}_{y}^{*}=-\mathcal{E}_{y}$. Thus, the longitudinal component $\mathcal{E}_{z}$ of the field in a guided normal mode is $\pi / 2$ out of phase with respect to the transverse components $\mathcal{E}_{x}$ and $\mathcal{E}_{y}$. This is a typical feature of transversely confined light fields [26-28,54]. 
It follows from the relations (A4) and Eqs. (A1), (A2), and (A8) that the field components of the odd $\mathcal{E}_{z}$-sine array modes satisfy the relations $\mathcal{E}_{x}(x,-y)=-\mathcal{E}_{x}(x, y)$, $\mathcal{E}_{z}(x,-y)=-\mathcal{E}_{z}(x, y), \quad \mathcal{E}_{y}(x, y)=-\mathcal{E}_{y}(-x, y), \quad$ and $\mathcal{E}_{z}(x, y)=-\mathcal{E}_{z}(-x, y)$, indicating the antisymmetry of $\mathcal{E}_{x}$ and $\mathcal{E}_{z}$ about the $x$ axis and that of $\mathcal{E}_{y}$ and $\mathcal{E}_{z}$ about the $y$ axis. It follows from these relations that, for the odd $\mathcal{E}_{z}$-sine array mode, the electric field at the two-fiber center $(x, y)=(0,0)$ is zero, that is, $\mathcal{E}(0,0)=0$. This feature of the odd $\mathcal{E}_{z}$-sine array mode can be used to produce a local minimum of a blue-detuned optical dipole potential to trap ground-state atoms [43-45] or a local minimum of a ponderomotive optical Rydberg-electron potential to trap Rydberg atoms $[55,56]$.
[1] L. Tong, R. R. Gattass, J. B. Ashcom, S. He, J. Lou, M. Shen, I. Maxwell, and E. Mazur, Nature (London) 426, 816 (2003).

[2] For a review, see T. Nieddu, V. Gokhroo, and S. Nic Chormaic, J. Opt. 18, 053001 (2016).

[3] For another review, see P. Solano, J. A. Grover, J. E. Homan, S. Ravets, F. K. Fatemi, L. A. Orozco, and S. L. Rolston, Adv. At., Mol., Opt. Phys. 66, 439 (2017).

[4] For a more recent review, see K. Nayak, M. Sadgrove, R. Yalla, F. Le Kien, and K. Hakuta, J. Opt. 20, 073001 (2018).

[5] J. P. Dowling and J. Gea-Banacloche, Adv. At., Mol., Opt. Phys. 37, 1 (1996).

[6] F. Le Kien, V. I. Balykin, and K. Hakuta, Phys. Rev. A 70, 063403 (2004).

[7] E. Vetsch, D. Reitz, G. Sague, R. Schmidt, S. T. Dawkins, and A. Rauschenbeutel, Phys. Rev. Lett. 104, 203603 (2010).

[8] A. Goban, K. S. Choi, D. J. Alton, D. Ding, C. Lacroûte, M. Pototschnig, T. Thiele, N. P. Stern, and H. J. Kimble, Phys. Rev. Lett. 109, 033603 (2012).

[9] C. Lacroûte, K. S. Choi, A. Goban, D. J. Alton, D. Ding, N. P. Stern, and H. J. Kimble, New. J. Phys. 14, 023056 (2012).

[10] J. B. Béguin, E. M. Bookjans, S. L. Christensen, H. L. Sørensen, J. H. Müller, E. S. Polzik, and J. Appel, Phys. Rev. Lett. 113, 263603 (2014).

[11] S. Kato and T. Aoki, Phys. Rev. Lett. 115, 093603 (2015).

[12] J. Lee, J. A. Grover, J. E. Hoffman, L. A. Orozco, and S. L. Rolston, J. Phys. B: At., Mol. Opt. Phys. 48, 165004 (2015).

[13] N. V. Corzo, B. Gouraud, A. Chandra, A. Goban, A. S. Sheremet, D. V. Kupriyanov, and J. Laurat, Phys. Rev. Lett. 117, 133603 (2016).

[14] V. I. Balykin, K. Hakuta, F. Le Kien, J. Q. Liang, and M. Morinaga, Phys. Rev. A 70, 011401(R) (2004).

[15] G. Sagué, A. Baade, and A. Rauschenbeutel, New J. Phys. 10, 113008 (2008).

[16] C. F. Phelan, T. Hennessy, and Th. Busch, Opt. Express 21, 27093 (2013).

[17] F. Le Kien and K. Hakuta, Phys. Rev. A 80, 013415 (2009).

[18] D. Reitz and A. Rauschenbeutel, Opt. Commun. 285, 4705 (2012).

[19] P. Schneeweiss, F. Le Kien, and A. Rauschenbeutel, New J. Phys. 16, 013014 (2014).

[20] M. Daly, V. G. Truong, C. F. Phelan, K. Deasy, and S. Nic Chormaic, New J. Phys. 16, 053052 (2014).

[21] M. Daly, V. G. Truong, and S. Nic Chormaic, Opt. Express 24, 14470 (2016).

[22] S.-P. Yu, J. D. Hood, J. A. Muniz, M. J. Martin, R. Norte, C.-L. Hung, S. M. Meenehan, J. D. Cohen, O. Painter, and H. J. Kimble, Appl. Phys. Lett. 104, 111103 (2014).

[23] A. Goban, C.-L. Hung, J. D. Hood, S.-P. Yu, J. A. Muniz, O. Painter, and H. J. Kimble, Phys. Rev. Lett. 115, 063601 (2015).
[24] J. D. Hood, A. Goban, A. Asenjo-Garcia, M. Lu, S.-P. Yu, D. E. Chang, and H. J. Kimble, Proc. Natl. Acad. Sci. USA 113, 10507 (2016).

[25] A. P. Burgers, L. S. Peng, J. A. Muniz, A. C. McClung, M. J. Martin, and H. J. Kimble, Proc. Natl. Acad. Sci. USA 116, 456 (2019).

[26] A. W. Snyder and J. D. Love, Optical Waveguide Theory (Chapman \& Hall, New York, 1983).

[27] D. Marcuse, Light Transmission Optics (Krieger, Malabar, 1989).

[28] K. Okamoto, Fundamentals of Optical Waveguides (Elsevier, New York, 2006).

[29] C. Ding, V. Loo, S. Pigeon, R. Gautier, M. Joos, E. Wu, E. Giacobino, A. Bramati, and Q. Glorieux, New J. Phys. 21, 073060 (2019).

[30] F. Le Kien, L. Ruks, S. Nic Chormaic, and T. Busch, New J. Phys. 22, 123007 (2020).

[31] W. Wijngaard, J. Opt. Soc. Am. 63, 944 (1973).

[32] E. Yamashita, S. Ozeki, and K. Atsuki, J. Lightwave Technol. 3, 341 (1985).

[33] N. Kishi, E. Yamashita, and H. Kawabata, J. Lightwave Technol. 7, 902 (1989).

[34] H. S. Huang and H. C. Chang, J. Lightwave Technol. 8, 945 (1990).

[35] C. S. Chang and H. C. Chang, J. Lightwave Technol. 15, 1213 (1997).

[36] H. S. Huang and H. C. Chang, Opt. Lett. 14, 90 (1989).

[37] C. S. Chang and H. C. Chang, J. Lightwave Technol. 15, 1225 (1997).

[38] M. L. Povinelli, M. Loncar, M. Ibanescu, E. J. Smythe, S. G. Johnson, S. Capasso, and J. D. Joannopoulos, Opt. Lett. 30, 3042 (2005).

[39] Z. Wang, S. Xie, X. Jiang, F. Babic, J. Huang, R. Pennetta, J. R. Koehler, and P. St. J. Russell, ACS Photonics 6, 2942 (2019).

[40] F. Le Kien, L. Ruks, S. Nic Chormaic, and T. Busch, New J. Phys. 23, 043006 (2021).

[41] I. H. Malitson, J. Opt. Soc. Am. 55, 1205 (1965).

[42] G. Ghosh, Handbook of Thermo-Optic Coefficients of Optical Materials with Applications (Academic, New York, 1997).

[43] S. Chu, Rev. Mod. Phys. 70, 685 (1998).

[44] C. Cohen-Tannoudji, Rev. Mod. Phys. 70, 707 (1998).

[45] W. D. Phillips, Rev. Mod. Phys. 70, 721 (1998).

[46] J. D. Jackson, Classical Electrodynamics, 3rd ed. (Wiley, New York, 1999).

[47] F. Le Kien, V. I. Balykin, and K. Hakuta, J. Phys. Soc. Jpn. 74, 910 (2005).

[48] F. Le Kien, P. Schneeweiss, and A. Rauschenbeutel, Eur. Phys. J. D 67, 92 (2013). 
[49] J. E. Sansonetti, W. C. Martin, and S. L. Young, Handbook of Basic Atomic Spectroscopic Data (version 1.00), National Institute of Standards and Technology, Gaithersburg, MD, http: //physics.nist.gov/Handbook.

[50] J. E. Hoffman, S. Ravets, J. A. Grover, P. Solano, P. R. Kordell, J. D. Wong-Campos, L. A. Orozco, and S. L. Rolston, AIP Adv. 4, 067124 (2014).

[51] J. P. Burke, S.-T. Chu, G. W. Bryant, C. J. Williams, and P. S. Julienne, Phys. Rev. A 65, 043411 (2002).

[52] M. Boustimi, J. Baudon, and J. Robert, Phys. Rev. B 67, 045407 (2003).
[53] M. C. Frawley, S. Nic Chormaic, and V. G. Minogin, Phys. Scr. 85, 058103 (2012).

[54] P. Lodahl, S. Mahmoodian, S. Stobbe, P. Schneeweiss, J. Volz, A. Rauschenbeutel, H. Pichler, and P. Zoller, Nature (London) 541, 473 (2017).

[55] S. K. Dutta, J. R. Guest, D. Feldbaum, A. WalzFlannigan, and G. Raithel, Phys. Rev. Lett. 85, 5551 (2000).

[56] D. Barredo, V. Lienhard, P. Scholl, S. de Léséleuc, T. Boulier, A. Browaeys, and T. Lahaye, Phys. Rev. Lett. 124, 023201 (2020). 